\title{
Scapular Retraction Under Adduction Load: an Alternative to Overhead Exercises to Activate Infraspinatus, Upper and Lower Trapezius in Subjects with and Without Shoulder Pain
}

\author{
Jefferson James dos Santos ${ }^{1}$, Rebeca Orozco Nagy ${ }^{1}$, Matheus Almeida de Souza ${ }^{1}$, Leonardo Intelangelo ${ }^{2}$, Michelle \\ Almeida Barbosa ${ }^{1}$, Gabriela Silveira-Nunes ${ }^{3}$, Alexandre Carvalho Barbosa1,* \\ 1 Department of Physical Therapy, Musculoskeletal Research Group - NIME, Federal University of Juiz de \\ Fora, Brazil, alexandre.barbosa@ufjf.edu.br \\ 2 Department of Physical Therapy, Musculoskeletal Research Unit - UIM, University Center for Assistance, \\ Teaching and Research - CUADI, Universidad del Gran Rosario - UGR, Rosario, Argentina, Leonardo.in- \\ telangelo@gmail.com \\ 3 Department of Medicine, Musculoskeletal Research Group - NIME, Federal University of Juiz de Fora, Bra- \\ zil, alexandre.barbosa@ufff.edu.br \\ * Correspondence: alexandre.barbosa@ufff.edu.br; Tel.: +55 339 9154-1851
}

\begin{abstract}
Exercises for lower trapezius (LT) often use overhead positions, causing compressive forces to the subacromial space. Scapular retraction would be an alternative to activate LT muscle. Electromyography of infraspinatus (IS), upper (UT) and LT was recorded during scapular retraction under progressive adduction loads of 42 participants, divided in 2 groups: with (SP, $\mathrm{n}=26$ ) and without shoulder pain (nSP, $n=16$ ). The adduction loads of $20,30,40$ and $50 \%$ of the maximal voluntary contraction were applied using a load cell. Normalized electromyography and the ratio between UT and LT (UT:LT) were used for statistical analysis. No differences were observed between groups, but a condition effect occurred for all muscles: UT showed higher values at $50 \%$ vs. $20 \%$ ( $p=0.004$ ); LT showed higher values on $40 \%$ and $50 \%$ ( $p=0.001 ; 0.006$ ). Higher values for IS were noted at $40 \%$ (vs $20 \%$; $\mathrm{p}=0.04$ ), and at $50 \%$ (vs. $20 \%$; $\mathrm{p}=0.001$, vs. $30 \%$, $\mathrm{p}=0.001$; vs. $40 \%$; $\mathrm{p}=0.001$ ). UT:LT showed lower values at $50 \%$ (vs. $20 \%$; $=0.001$, and vs. $30 \%$; $p=0.016$ ). Scapular retraction with adduction loads at $40-50 \%$ is an alternative to overhead exercises aiming to activate the LT and the IS muscles. The exercise ensures higher levels of LT and IS excitation, without increasing UT excitation.
\end{abstract}

Keywords: Shoulder impingement syndrome, Scapula, Exercise therapy, Exercise movement techniques, Musculoskeletal pain

\section{Introduction}

Shoulder pain is one of the most common musculoskeletal complaints, affecting 4.7$46.7 \%$ of the adult population, and its prevalence increases with age ${ }^{1-3}$. A painful shoulder causes mobility restrictions, functional impairments in daily activities, work disability, and a relevant impact to public health services 1,2 . The shoulder pain can last for weeks to months and become a recurrent condition ${ }^{3,4}$. Moreover, aging populations, and consequent longer careers might increase the burden of shoulder pain ${ }^{5}$. Impingement syndrome is a term for injury of structures in the subacromial space ${ }^{6-8}$. It has the highest prevalence among shoulder issues, accounting for $36 \%$ of shoulder disorders 9,10. Many shoulder symptoms have been associated with abnormal excitation of the scapular and rotator cuff muscles ${ }^{11,12}$. A systematic review aimed to summarize the current evidence regarding scapulothoracic muscle activity based on surface electromyography in patients with shoulder impingement symptoms or glenohumeral instability vs healthy controls ${ }^{13}$. The authors concluded that impingement syndrome patients present an overactive upper trapezius and underactive lower trapezius and serratus anterior in contrast to healthy 
controls. The majority of the included studies showed higher upper trapezius muscle activity during abduction in the scapular plane and in the frontal plane in the impingement syndrome group compared to the control group. For the lower trapezius and serratus anterior, decreased muscle activity was observed in the impingement syndrome group. Prior studies also showed altered movement patterns, including decreased scapular external rotation, increased or decreased scapular upward rotation, and decreased scapular posterior tilting ${ }^{12,14}$. A study reported decreased activity of the infraspinatus muscle in the range of $30^{\circ}$ to $90^{\circ}$ of flexion in the scapular plane, concluding that the deficit in the depression of the humeral head can lead to subacromial friction ${ }^{15}$. It also has been reported that subjects with unilateral subacromial impingement syndrome have decreased infraspinatus muscle activity during external rotation ${ }^{16}$.

From a therapeutic perspective, physiotherapy represents one of the primary options for shoulder pain treatment. There is growing evidence of the importance of active exercise therapy in the treatment of shoulder pain 1,3,17. Optimal exercises for shoulder rehabilitation need to recruit lower trapezius and rotator cuff muscles with concomitant minimal activity of the upper trapezius, as excessive activity of this last muscle might increase the anterior tilt of the scapulothoracic joint 18,19 . Therefore, the shoulder rehabilitation program should include exercises focused on the activation of upward rotators (serratus anterior, upper and lower trapezius) while minimizing the activation of the scapular downward rotators (latissimus dorsi, levator scapulae, rhomboids, and the pectoralis major and minor muscles) ${ }^{20}$ to increase the subacromial space during the overhead movements of the arm, and implement effective interventions. A study ${ }^{21}$ found a decreased in the electromyographic activity of the anterior deltoid and infraspinatus muscles, in 58 individuals with subacromial impingement syndrome between 24 and 85 years with an average of 40 months of pain. Those decreased activities occurred as an adaptive strategy to avoid pain. However, there is no consensus as to the most effective exercise strategy 9,17,22. Several types of exercises have been proposed, but little data is available to guide the physical therapist in selecting the most appropriate protocol 3,12,17,23,24. In fact, most of the proposed exercises use an overhead positioning. $19,23,25,26$ This position might potentially cause compressive forces to the subacromial space 27,28 . Even in healthy people, the overhead exercises like in wall push-up plus might place the scapula in a position potentially associated with shoulder impingement ${ }^{29}$. Due to shoulder kinematics during a wall push-up plus, such exercise should be cautiously considered during early shoulder rehabilitation, especially in patients with subacromial impingement and/or shoulder pain.

Previous findings showed that subjects with previous shoulder injury have lower activation of the lower trapezius muscle excitation compared to controls and to contralateral limb ${ }^{30}$. It was originally suggested that global muscle weakness was the main factor for shoulder and scapular problems. However, recent research showed that muscular imbalance may contribute as well ${ }^{31}$. Theoretically, compensation may occur through increased excitation of the upper trapezius muscle combined with decreased excitation and control of the lower trapezius muscle ${ }^{32}$. A study ${ }^{19}$ found an increased upper trapezius activity with reduced activation of lower trapezius secondary to shoulder elevation even in retracted scapular position, which lead to consequent higher upper-lower trapezius muscle ratios (UT:LT). This finding suggests the existence of fine activity coordination between the trapezius portions to not counteract the action of the upper trapezius muscle 19. Thus, an exercise combining low levels of upper trapezius muscle excitation, moderate to high levels of lower trapezius muscle and rotator cuff muscles excitation, and subacromial non-compressive positioning would be very beneficial for treating subjects with shoulder pain. Accordingly, previous reports and researchers have established a scientific framework that justifies the application of specific exercises in this population.

Therefore, the present study primarily aimed to assess the excitation levels of infraspinatus, upper trapezius and lower trapezius muscles during a scapular retraction exercise under progressive adduction loads in subjects with and without painful shoulder. 
A secondary objective was to analyze which load level of resisted adduction would generate the best ratio between upper and lower trapezius muscles. The hypothesis is that the lower trapezius and infraspinatus would present progressive higher levels of muscle excitation during scapular retraction for both groups while the adduction load increases, without any significant increases in upper trapezius muscle excitation nor between-group differences. An additional hypothesis is that the higher levels of adduction loads would generate the desired ratio levels of upper and lower trapezius muscle excitation when compared to lower levels of adduction loads.

\section{Materials and Methods}

\subsection{Participants}

Forty-two subjects participated in this study (Table 1). The subjects were recruited by public invitation through folders and personal contacts. They were divided into 2 groups, according to presence of shoulder pain: with (Painful Shoulder group) and without shoulder pain (non-Painful Shoulder group). The subjects were assessed in the biomechanical laboratory facilities on the Federal University of Juiz de Fora - Campus Governador Valadares (Minas Gerais, Brazil). All assessments were provided onsite. The objectives of the study were explained to the subjects, and they were notified of the benefits and potential risks involved before signing an informed consent form before participation. The ethics committee for the human investigation of the Federal University of Juiz de Fora approved the procedures employed in the study (protocol number 25614019.4.0000.5147).

Table 1. Participant's characteristics

\begin{tabular}{lccc}
\hline Outcome & SP Group & nSP Group & p \\
\hline n (male/female) & $26(7 / 19)$ & $16(5 / 11)$ & $0.630^{\mathrm{a}}$ \\
Age (years) & $26 \pm 8$ & $22 \pm 2$ & $0.138^{\mathrm{b}}$ \\
Weight (Kg) & $67 \pm 12$ & $57 \pm 10$ & $0.018^{\mathrm{b}}$ \\
Height (m) & $1.69 \pm 8$ & $1.66 \pm 10$ & $0.286^{\mathrm{b}}$ \\
BMI (Kg/m²) & $23 \pm 5$ & $21 \pm 5$ & $0.036^{\mathrm{b}}$ \\
Diskinesis (yes/no [\%]) & $9 / 91$ & $7 / 93$ & $0.837^{\mathrm{a}}$ \\
QuickDASH score & $25 \pm 3$ & $2 \pm 1.7$ & $>0.001^{\mathrm{c}}$ \\
SPADI-total & $31 \pm 16$ & $2.4 \pm 0.5$ & $>0.001^{\mathrm{c}}$ \\
SPADI-pain & $40 \pm 4.4$ & $3.6 \pm 1.9$ & $>0.001^{\mathrm{c}}$ \\
\hline
\end{tabular}

Legend: $\mathrm{SP}$ = with shoulder pain; $\mathrm{SSP}$ = without shoulder pain; ${ }^{a}$ Chi-square test; bStudent's independent t-test; cMann-Whitney test

Participants were considered healthy if they exhibited a full, pain-free range of motion, no symptom during the physiotherapy assessments. Individuals in both groups were excluded if they had cervical spine-related symptoms, a history of dislocations or shoulder surgery, cortisone injections within the last six months, systemic and neurological reported diseases, adhesive allergies, and history of shoulder fracture or surgery. The subjects were asked to avoid participation in an unusually strenuous activity $48 \mathrm{~h}$ before the testing session. All subjects complied with the orientation.

The Shoulder Pain and Disability Index (SPADI - Brazilian Portuguese version) ${ }^{33}$ and the quick version of the Disabilities of the Arm, Shoulder and Hand Questionnaire (QuickDASH - Brazilian Portuguese version $)^{34}$ were used to assess the extent of symptoms in the involved shoulder, and as additional scores to classify the subjects into both groups. The QuickDASH uses a 5-point Likert scale for answers to questions focused on the level of difficulty of certain tasks, how severe the pain is, and how the disability affects social and 
work/sport activities. A higher score indicates the worse function, with the highest possible score equal to 100. The SPADI was developed to measure current shoulder pain and disability in an outpatient setting. It contains 13 items to assess 2 domains: a 5-item subscale to assess pain and an 8-item subscale that measures disability. The definition of shoulder pain includes recent pain ( $>1$ week) near to anterolateral shoulder region. Patients were included in the SP group if they reported chronic shoulder pain $(>1$ month during the last year, with a minimum pain intensity of 3/10 on the Numeric Rating Scale) in the anterior deltoid region of their dominant shoulder and if at least 3 of the following criteria were positive: (1) Positive Neer sign, designed to reproduce symptoms of rotator cuff impingement through flexing the shoulder and pressure application; (2) Positive Hawkins sign, designed to indicate impingement of all structures located between the greater tubercle of the humerus and the coracohumeral ligament. The seated subject flex the shoulder and the elbow to 90 degress while the examiner supports proximal to both the patient's wrist and elbow to ensure maximal relaxation. Then, the examiner rotates the patient's arm internally; (3) Positive Jobe's sign, which is performed by the examiner passively elevating the patient's shoulder to 90 degrees of abduction with internal rotation. The examiner then applies a downward pressure against the arm. The presence of pain or abnormal weakness is considered positive; (4) Painful Arc, the patient abducts the arm in the scapular plane. The test is considered positive if the patient experiences pain in the range of 60 to 120 degrees of abduction which reduces once past 120 degrees of abduction; and (5) Positive Resistance Test against External Rotation, which assesses the infraspinatus and teres minor muscles. The elbow is flexed at 90 degrees and in slight abduction, while the arm is passively externally rotated to its maximum ${ }^{35}$.

\subsection{Instruments}

An acquisition module with eight analog channels (Miotec ${ }^{\mathrm{TM}}$, Biomedical Equipments, Porto Alegre, RS, Brazil) continuously recorded the biological signals. The conversion from analog to digital signals was performed by an A/D board with a 14-bit resolution input range, the sampling frequency of $2 \mathrm{kHz}$, a common rejection module greater than $100 \mathrm{~dB}$, signal-noise ratio less than $3 \mu \mathrm{V}$ Root Mean Square and impedance of $109 \Omega$. The collected data was windowed at $125 \mathrm{~ms}$ using the Miotec ${ }^{\mathrm{TM}}$ Suite Software. The sEMG signals were recorded in root mean square in $\mu \mathrm{V}$ with surface Maxicor ${ }^{\mathrm{TM}}$ (Maxicor Medical Products, Paraná, Brazil) Ag/AgCl electrodes with a radius of $1 \mathrm{~cm}$ and center-to-center distance of $2 \mathrm{~cm}$, applied in a transverse orientation parallel to the underlying fibers on a muscle site. A reference electrode was placed on the left lateral humeral epicondyle. Surface electromyography signals were amplified and filtered (Butterworth fourth-order, 20-450 Hz bandpass filter, $60 \mathrm{~Hz}$ notch filter). Before electrode placement, the skin was cleaned with $70 \%$ alcohol, followed by an exfoliation using specific sandpaper for skin and a second cleaning with alcohol. The electrodes were positioned on the upper and lower trapezius according to Surface Electromyography for Non-Invasive Assessment of Muscles (SENIAM - http://seniam.org/shoulder_location.htm). SENIAM does not provide electrode placement recommendations for infraspinatus muscle. Therefore, we followed the electrode placement recommendations and descriptions by a previous study ${ }^{36}$. The electrodes were placed on the upper trapezius at $50 \%$ on the line from the acromion to the spine on vertebra C7. For the lower trapezius, the electrodes were placed at $2 / 3$ on the line from the trigonum spinea to the 8 th thoracic vertebra. Finally, for the infraspinatus muscle the electrodes were placed $4 \mathrm{~cm}$ below the spine of the scapula, over the infrascapular fossa of the scapula, laterally, but not over the posterior deltoid muscle.

A calibrated load cell (Miotec ${ }^{\mathrm{TM}}$, Biomedical Equipments, Porto Alegre, RS, Brazil; maximum tension-compression $=200 \mathrm{kgf}$, the precision of $0.1 \mathrm{kgf}$, the maximum error of measurement $=0.33 \%$ ) was used to control the levels of adduction loads. The validity of the load cell was previously assessed ${ }^{37}$. The load cell was coupled and synchronized with the acquisition module. Two straps were used to handle the load cell. Both were connected to the load cell (Figure 1A). The $1^{\text {st }}$ strap was positioned on the subject's distal arm (right 
above the humeral epicondyles' line). The $2^{\text {nd }}$ strap was used by the rater to control the level of load applied in each condition. All pieces of information were recorded and processed offline using the software Miotec Suite ${ }^{\mathrm{TM}}$ (Miotec Biomedical Equipments, Porto Alegre, RS, Brazil).

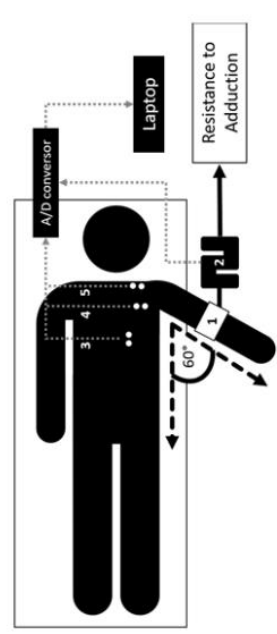

(a)

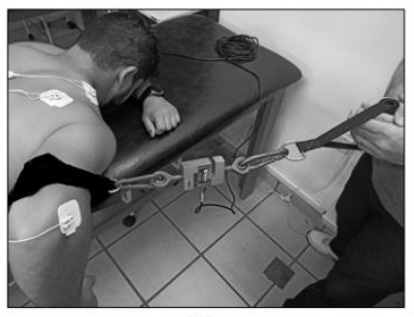

(b)

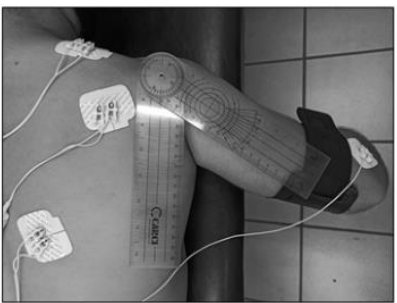

(c)

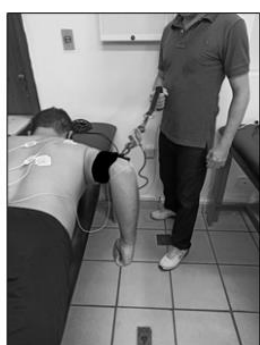

(d)

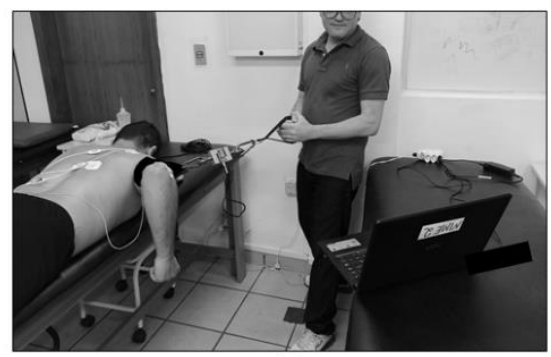

(e)

Figure 1. Procedure for data recording. Subject in prone (1A and B), leaving the arm out of bed in 60 degrees of abduction (1C). The strap positioned right above the humeral epicondyles' line (1C). The rater and participant's initial positioning (1D). Scapular retraction while the rater controls the level of adduction load using the biofeedback software (1E). Legend (1A): 1. Strap; 2. Load cell; 3. Surface electromyography sensor (sEMGs) positioning - lower trapezius; 4. sEMGs positioning - infraspinatus; 5. sEMGs positioning - upper trapezius.

\subsection{Maximal Voluntary Isometric Contraction for Electromyography Normalization Purposes}

Three 5-second maximal voluntary isometric contractions (with 3 minutes of rest between each trial) from each muscle were performed by each participant. For each tested muscle, a rest of 5 minutes was allowed. Standardized verbal commands ("start", "force", "stop") were used by the same rater for all test recordings. Before recording the first maximal voluntary isometric contractions trial, the participants received an explanation about the positioning and the direction they should exert maximal effort. Then, a 5-second familiarization trial followed by 3 minutes of rest was performed to verify the participants' understanding of the procedures. The mean among the maximal voluntary isometric contractions trials was used to normalize the electromyographic signals. For the infraspinatus and upper trapezius muscles, the participant remained standing. For the infraspinatus, the shoulder was externally rotated with the elbow flexed at $90^{\circ}$. The participant was requested to perform an isometric contraction of shoulder external rotators against the rater's manual resistance ${ }^{36}$. For the upper trapezius muscle, the patient resisted abduction with the arm placed in 90 degrees of shoulder abduction, the elbow flexed 90 degrees 38 . For the lower trapezius muscle, the participant was asked to lie in the prone position. The maximal voluntary isometric contraction was collected with the shoulder fully extended with the scapula maximally retracted, while the resistance was applied against the fully flexed elbow on the distal humerus. The participant was instructed to resist against the downward force. The scapular retraction was chosen due to its capacity to activate specific 
sEMG patterns in the lower trapezius muscle. ${ }^{39}$ Participant's positioning was monitored by the researcher to minimize compensatory movements.

\subsection{Maximal Voluntary Isometric Contraction to Control the Adduction Resistance}

Three 5-second maximal voluntary isometric contractions (with 3 minutes of rest between trials) in adduction were recorded using the load cell. The subject was instructed to lie prone, leaving the arm to be evaluated out of bed. The therapist positioned the strap on the distal third of each subject's arm (right above the humeral epicondyles' line). The subject was instructed to maximally pull the load cell through the attached strap for 5seconds. Standardized verbal commands ("start", "force", "stop") were used by the same rater for all tests' recordings.

\subsection{Exercise description}

The subject was instructed to lie prone, leaving the arm to be evaluated out of bed parallel to the floor (Figure 1B). All sensors for electromyography were previously positioned. The therapist positioned the strap on the distal third of the subject's arm right above the line between the humeral epicondyles. Also, the magnitudes of progressive resistance were percentages of the individual maximal voluntary isometric contraction in adduction. After 10 seconds of initial rest, resisted adductions at 60 degrees (measured by goniometer - Figure 1C) were performed for 10 seconds at $20 \%, 30 \%, 40 \%$ and $50 \%$ of the previously collected adduction maximal voluntary isometric contraction with 20 seconds of rest between tasks (Figure 1E and 2). As the participant was lied in prone, the rater asked the participant to actively adduct the arm. When the chosen level was achieved, the therapist asked the participant to hold the contraction (Figure 2). Concomitantly, the subject was instructed to perform maximal scapular retraction of the evaluated shoulder (Figure 1E). The level of resistance was applied by the therapist as previously set and realtime controlled using the Biotrainer ${ }^{\mathrm{TM}}$ visual biofeedback software (Miotec ${ }^{\mathrm{TM}}$, Biomedical Equipment, Porto Alegre, RS, Brazil - Figure 2). The Excel software was used to randomize the trials' order. To prevent fatigue, only 1 set of trials was performed. To ensure the absence of muscle fatigue, the median frequency was obtained using the real-time feedback software and monitored during the entire protocol. The median frequency from the $50 \%$ and the $20 \%$ were extracted to check if there were differences between them. All assessments were provided onsite. No practice at home was asked. Attendance at the session was, therefore, taken as compliance with the assessment protocol. No co-interventions were performed in either group, and no adverse effects were reported by any participant during the assessments.

\subsection{Data extraction}

All data were offline extracted using the Miotec Suite ${ }^{\mathrm{TM}}$ Software (Miotec ${ }^{\mathrm{TM}}$, Biomedical Equipments, Porto Alegre, RS, Brazil). As the load cell was synchronized with the electromyography channels, the trained rater set the interval using the force onset. Three 1 -s windows of the initial rest were collected (first 10 seconds of rest), and the force onset was defined by three times the standard deviation from the averaged rest intervals plus the mean itself. The interval started when the signal exceeded the onset threshold value. Conversely, the end of the interval was set using the same threshold. The interval means were used for statistical analysis (mean muscle excitation). The ratio between the upper and lower trapezius muscles was also calculated by dividing the normalized electromyographic mean obtained from the upper trapezius by the obtained from the lower trapezius 40.

\subsection{Statistics}


Data were presented as means and standard deviation. The mixed factorial Analysis of Variance (ANOVA) with repeated measures was used to rate differences within- and between-group. The factor group* condition was also assessed. The condition is defined as the level of adduction load applied during the protocol. All data was reworked using the Holm's post hoc test to compare differences among trials $(20 \%, 30 \%, 40 \%$ and $50 \%$ of maximal adduction contraction) avoiding pairwise multiple comparisons. The significance was set at $\mathrm{p}<0.05$. The standardized differences for the comparisons were analyzed using the partial eta square's effect size $(\eta 2 p)$. The magnitude of the $\eta 2 p$ was qualitatively interpreted using the following thresholds: $~ 0.01$ (small), $>0.09$ (medium) and $>0.25$ (large). All analysis was performed using the JAMOVI software (Version 0.9.6, JAMOVI project, 2019).

\section{Results}

Descriptive and inferential data are presented in table 2. No differences were observed between $50 \%$ and $20 \%$ median frequency values during the protocol $(p>0.05)$, ensuring no influence of fatigue. Both groups presented similar patterns of muscle activity, with low levels of excitation of the upper trapezius muscle. No between-group differences were observed for the upper trapezius muscle $(F=0.39 ; p=0.537 ; \eta 2 p=0.012)$, the lower trapezius muscle $(\mathrm{F}=0.14 ; \mathrm{p}=0.294 ; \eta 2 \mathrm{p}=0.033)$, the infraspinatus muscle $(\mathrm{F}=1.96$; $\mathrm{p}=0.171 ; \eta 2 \mathrm{p}=0.058)$, and for the ratio UT:LT $(\mathrm{F}=1.82 ; \mathrm{p}=0.186 ; \eta 2 \mathrm{p}=0.052)$.

Table 2. Muscle excitation at different adduction loads (in \% of the maximal voluntary isometric contraction using the load cell).

\begin{tabular}{|c|c|c|c|c|c|}
\hline Muscle & $\begin{array}{c}\text { Adduction } \\
\text { Load }\end{array}$ & $\begin{array}{l}\text { Painful } \\
\text { Shoulder } \\
\text { Group (\%) }\end{array}$ & $\begin{array}{c}\text { Non-Painful } \\
\text { Shoulder } \\
\text { Group (\%) }\end{array}$ & $\begin{array}{l}\text { Between- } \\
\text { group } \\
\text { (F;p) }\end{array}$ & $\begin{array}{l}\text { Within-group pairwise } \\
\text { comparison for time effect } \\
\text { (p value) }\end{array}$ \\
\hline & $20 \%$ & $6 \pm 4$ & $6 \pm 5$ & \multirow{4}{*}{$0.39 ; 0.537$} & \multirow{4}{*}{$20 \%<50 \%(p=0.004)$} \\
\hline Upper & $30 \%$ & $8 \pm 7$ & $6 \pm 5$ & & \\
\hline \multirow[t]{2}{*}{ Trapezius } & $40 \%$ & $7 \pm 6$ & $6 \pm 3$ & & \\
\hline & $50 \%$ & $9 \pm 7$ & $7 \pm 4$ & & \\
\hline \multirow{4}{*}{$\begin{array}{c}\text { Lower } \\
\text { Trapezius }\end{array}$} & $20 \%$ & $34 \pm 16$ & $49 \pm 29$ & \multirow{4}{*}{$1.14 ; 0.294$} & $20 \%<40 \%(p=0.001)$ \\
\hline & $30 \%$ & $46 \pm 26$ & $54 \pm 35$ & & $20 \%<50 \%(p=0.001)$ \\
\hline & $40 \%$ & $58 \pm 40$ & $66 \pm 37$ & & $30 \%<40 \%(p=0.006)$ \\
\hline & $50 \%$ & $72 \pm 44$ & $87 \pm 42$ & & $30 \%<50 \%(p=0.001)$ \\
\hline \multirow{4}{*}{ Infraspinatus } & $20 \%$ & $23 \pm 14$ & $18 \pm 6$ & \multirow{4}{*}{$1.96 ; 0.171$} & $20 \%<40 \%(p=0.040)$ \\
\hline & $30 \%$ & $25 \pm 17$ & $17 \pm 6$ & & $20 \%<50 \%(p=0.001)$ \\
\hline & $40 \%$ & $29 \pm 21$ & $21 \pm 6$ & & $30 \%<50 \%(p=0.001)$ \\
\hline & $50 \%$ & $37 \pm 27$ & $27 \pm 8$ & & $40 \%<50 \%(\mathrm{p}=0.001)$ \\
\hline \multirow{4}{*}{ UT:LT } & $20 \%$ & $0.22 \pm 0.18$ & $0.16 \pm 0.13$ & \multirow{4}{*}{$1.82 ; 0.186$} & \\
\hline & $30 \%$ & $0.22 \pm 0.20$ & $0.14 \pm 0.08$ & & $20 \%<50 \%(p=0.001)$ \\
\hline & $40 \%$ & $0.18 \pm 0.15$ & $0.13 \pm 0.11$ & & $30 \%<50 \%(\mathrm{p}=0.016)$ \\
\hline & $50 \%$ & $0.16 \pm 0.14$ & $0.10 \pm 0.06$ & & \\
\hline
\end{tabular}


Legend: UT:LT = ratio between upper and lower trapezius muscles.

Progressive higher levels of the lower trapezius and infraspinatus excitation during scapular retraction. A progressive effect for adduction load was observed (table 2) at within-group analysis (upper trapezius: $\mathrm{F}=4.19 ; \mathrm{p}=0.008 ; \eta 2 \mathrm{p}=0.11$ / lower trapezius: $\mathrm{F}=32.7 ; \mathrm{p}=0.001 ; \eta 2 \mathrm{p}=0.49$ / infraspinatus: $\mathrm{F}=19.09 ; \mathrm{p}=0.001 ; \eta 2 \mathrm{p}=0.37)$. The upper trapezius pairwise comparison showed higher values at $50 \%$ compared to $20 \%$ of maximal adduction load, but always below $20 \%$ of the maximal voluntary isometric contraction. For the lower trapezius, higher values were observed on $40 \%$ and $50 \%$ of the maximal adduction load, with differences as follows: $20 \%<40 \%, 20 \%<50 \%, 30 \%<40 \%, 30 \%<50 \%$, and $40 \%$ $<50 \%$. For infraspinatus, higher values at $40 \%$ and $50 \%$ of the maximal adduction load differences were noted as follows: $20 \%<40 \%, 20 \%<50 \%, 30 \%<50 \%$, and $40 \%<50 \%$.

Higher levels of adduction loads generated lower ratio levels of upper and lower trapezius muscle excitation (table 2). An effect was observed for UT:LT ratio comparing $20 \%$ vs. $50 \%$, and $30 \%$ vs. $50 \%(\mathrm{~F}=5.83 ; \mathrm{p}=0.001 ; \eta 2 \mathrm{p}=0.15)$. The UT:LT ratio at $50 \%$ was significantly lower than $20 \%$ and $30 \%$.

\section{Discussion}

The primary focus of this study was to investigate the excitation of infraspinatus, upper trapezius, and lower trapezius muscles during the scapular retraction exercise while progressive adduction loads were controlled by biofeedback. The results showed no differences between Painful Shoulder and non-Painful shoulder groups, but there was an effect among distinct levels of adduction loads. The absence of between-group differences constitutes an important finding, as it suggests the benefits of the current exercise in accomplishing the exercise's goals for both groups meaning that individuals with shoulder pain were able to achieve the same levels of muscle excitation on each level of adduction load compared to those without pain during the scapular retraction.

A study assessed the excitation levels of upper and lower trapezius, among other muscles, during shoulder shrugging and retraction exercises ${ }^{20}$. The findings showed low to moderate levels of excitation for upper trapezius (25-38\% of maximal voluntary isometric contraction) with even lower levels for lower trapezius (3-22\% of maximal voluntary isometric contraction). Also, the exercises were all performed in a potential compressive position. The exercise proposed in the current study would potentially avoid subacromial compression due to non-overhead positioning 18,19 , with additional advantages in targeting the lower trapezius muscle excitation concomitant to lower upper trapezius excitation. Such type of exercise would be an option for exercise prescription aiming the success of the therapy ${ }^{41}$. Another study assessed the levels of scapular muscles during scapula retraction exercises at various shoulder abduction angles ${ }^{18}$. The authors reported low levels of upper trapezius excitation (6-18\% of maximal voluntary isometric contraction) and moderate middle trapezius recruitment (22-31\% of maximal voluntary isometric contraction). However, the lower trapezius muscle was not assessed. Muscle activation below $20 \%$ is considered clinically low, and activity between $20 \%$ and $40 \%$ is considered clinically moderate ${ }^{39,42}$. An important purpose of the exercises for shoulder rehabilitation is to recruit lower trapezius and rotator cuff muscles with concomitant minimal activity of the upper trapezius, as excessive activity of this last muscle might increase the anterior tilt of the scapulothoracic joint ${ }^{18,19}$, leading to compensations. To our knowledge, there is no report in the literature of an exercise addressing combined higher excitation of the lower trapezius and infraspinatus muscles without increasing the upper trapezius excitation, and potentially minimizing the impact without deteriorating the subacromial space or exacerbating the painful condition. A study assessed the effects of scapular retraction-pro- 
traction position with scapular elevation on shoulder girdle muscle activity during glenohumeral abduction ${ }^{43}$. The lower trapezius activity was significantly higher in retracted scapular position than in neutral and protracted positions, both with and without scapular elevation. However, the lower trapezius activity was significantly lower with scapular elevation (i.e., with concomitant upper trapezius muscle contraction) than without during retracted scapular position. The authors suggested the existence of a fine trapezius coordination pattern between the muscle portions to not counteract the action of the upper trapezius muscle and promote smooth scapular movements.

The present results suggest a pattern of muscle excitation that, in opposition to upper trapezius recruitment, the shoulder adduction lead to progressive higher values of lower trapezius excitation combined with very low values of upper trapezius muscle activity using the concomitant progressive adduction loads. A study had similar findings using the infraspinatus and the deltoid muscles ${ }^{44}$. The humerus was actively adducted against a sphygmomanometer (using $80 \%$ maximum force output) while maximal effort external rotation was manually resisted. The results showed a reduced posterior deltoid electromyographic activity during the active adduction compared with no resisted adduction, without reducing the activity of the infraspinatus.

Previous studies showed that moderate levels of muscle activation $(20-40 \%$ of the maximal voluntary isometric contraction) are considered adequate to retrain neuromuscular control of the scapular girdle ${ }^{39,45}$. As the upper trapezius excitation showed a low level of muscle activation (range between 6-9\% of maximal voluntary isometric contraction) with moderate to high levels of the lower trapezius excitation (range between 34$87 \%$ of maximal voluntary isometric contraction), further studies may consider the role of the present exercise in a neuromuscular training protocol. Moreover, the upper trapezius muscle excitation is low enough and lower trapezius muscle excitation is high enough to potentially cause effects on prospective training, as many authors have proposed exercises that emphasize the activation of the lower trapezius with less activation of the upper trapezius to improve the neuromuscular control of the scapula, and to prevent and treat people with shoulder pain. ${ }^{39,46}$ Altered patterns of muscle excitation may represent attempts to minimize muscle activity during painful movements. The altered neural control, muscle weakness, and muscle imbalance are factors that account for the reduction in muscle excitation ${ }^{11,30}$. The increased level of lower trapezius activity combined with very low upper trapezius recruitment found in the present exercise may minimize the compensatory upper trapezius muscle activity usually found in patients with a painful shoulder ${ }^{19}$. Also, the high achieved levels of lower trapezius muscle excitation can be potentially useful to contribute to strength and coordination training. In an interesting study, the activity levels of the upper trapezius, middle trapezius, and lower trapezius muscles were measured during various tasks and selectively activated each of the muscles ${ }^{47}$. The authors found that the patients with shoulder impingement syndrome were unable to activate middle trapezius and lower trapezius muscle as highly as healthy individuals. Another study investigated differences in muscle excitation by comparing previously injured limb of individuals with a history of glenohumeral joint injury and healthy matched controls during functional isometric contractions ${ }^{30}$. In the previously injured group, the involved lower trapezius muscle was significantly less activated than the same muscle of the uninvolved limb during scaption and abduction.

The UT:LT ratio results showed progressively lower values at $40 \%$ and $50 \%$ of maximal adduction load compared to $20 \%$ and $30 \%$. Lower values of UT:LT ratio indicate the desirable scenario with higher lower trapezius excitation combined with lower upper trapezius excitation. The UT:LT ratio has been considered an important factor as the scapular muscles do not work in isolation, but synergistically to produce a controlled scapular movement with consequent shoulder stability 18,47,48. Previous studies have assessed scapular muscle excitation and reported higher UT:LT ratio in those with shoulder impinge- 
ment syndrome compared with healthy controls 18,19 . The scapular motion may be disrupted during dynamic arm activities when the activity between the scapulothoracic muscles is not coordinated ${ }^{19,40}$. A study showed a disruption in muscle activity ratios between sections of the trapezius and between the trapezius and serratus anterior during an active arm elevation task in patients with subacromial pain syndrome, highlighting the lower trapezius muscle as an important part of altered ratios during arm ascent and descent ${ }^{40}$.

The present sample was mainly constituted by adult young women. A narrative review showed that the estimated prevalence of shoulder pain was higher in women (15.4\% in men and $24.9 \%$ in women) who reported weekly episodes of pain ${ }^{49}$. Another study retrospectively assessed painful shoulders from medical files, finding a prevalence of women $(66.21 \%)^{50}$. Additionally, severe pain is more frequently reported in women (56.1\%) compared to men $(25.0 \%)^{51}$. A longitudinal study assessed the risk factors for neck and shoulder pain among young adults in the transition from technical school to working life ${ }^{52}$. The authors reported that high mechanical workload was associated with neck and shoulder pain in women.

In terms of practical applications, the healthy controls and subjects with shoulder pain showed similar upper trapezius, lower trapezius, and infraspinatus muscle excitation during scapular retraction exercise with progressive adduction loads, suggesting that the exercise could be potentially used in strengthening programs without leading abnormal trapezius muscle activity in subjects with shoulder pain. An important topic is that no load was applied specifically against the retraction movement, but only counteracting the adduction. Thus, a greater level of excitation could be achieved in all scapular muscle retractors by directing loads in other planes. However, further prospective studies need to clarify the utility of the exercise into a rehabilitation training program. Also, a distinct study design may elucidate the level of muscle excitation with additional resistance applied to scapular retraction.

Limitations of the present study must be addressed. Distinct shoulder angles may elicit different levels of excitation for all muscles, as the present study did not assess the shoulder full range of motion. While this study provided useful information regarding the muscles being activated during various levels of adduction loads, no scapular kinematics was documented due to technical issues (e.g., absence of 3D analysis), as the laboratory did not have a system to acquire 3D movement data. Additional information about scapular movements, along with the others scapular girdle and rotator cuff muscles excitation would provide data to select exercises based on the subject's needs. Another important limitation is the limited number of muscles assessed by the present study. Other muscle interactions may be elicited with complementary electromyographic analysis. The convenience sample was also constituted by young adults and mostly by women. The results may vary in different ranges of age, as well as in other gender proportion.

\section{Conclusions}

The present results support the use of $40-50 \%$ adduction loads to ensure higher levels of lower trapezius and infraspinatus excitation, without increasing upper trapezius muscle excitation. The scapular retraction exercise under 40 and $50 \%$ of maximal voluntary isometric contraction adduction loads is an option to ensure increased levels of excitation of infraspinatus and lower trapezius, with low levels of upper trapezius muscle excitation, which is an alternative to overhead exercises aiming to activate the lower trapezius and the infraspinatus muscles.

Author Contributions: Conceptualization, A.C.B., G.S.N. and M.A.B.; methodology, A.C.B. and J.J.S.; formal analysis, A.C.B.; investigation, R.O.N., M.A.S. and J.J.S.; resources, L.I.; data curation, 
L.I. and A.C.B.; writing - original draft preparation, L.I., J.J.S. and A.C.B; writing-review and editing, A.C.B. and G.S.N.; supervision, M.A.B and A.C.B; project administration, A.C.B.; funding acquisition, A.C.B. All authors have read and agreed to the published version of the manuscript.

Funding: This study was supported in part by the Coordenação de Aperfeiçoamento de Pessoal de Nível Superior - Brasil (CAPES) - Finance Code 001, and by the Fundação de Amparo à Pesquisa de Minas Gerais (FAPEMIG). This study was also financially supported by the Institutional Extension Scholarship Program (PIBEX).

Institutional Review Board Statement: The ethics committee for the human investigation of the Federal University of Juiz de Fora approved the procedures employed in the study (protocol number 25614019.4.0000.5147).

Informed Consent Statement: Informed consent was obtained from all subjects involved in the study.

Data Availability Statement: Data Availability Statement: The data presented in this study are openly available in Barbosa, Alexandre (2021), "Scapular retraction under adduction load: an alternative to overhead exercises to activate infraspinatus, upper and lower trapezius in subjects with and without shoulder pain", Mendeley Data, V1, doi: 10.17632/2sv5hbdz4s.1.

Acknowledgments: Special thanks to UFJF-GV Department of Physical Therapy and to the Diretoria de Relações Internacionais - UFJF.

Conflicts of Interest: The authors declare no conflict of interest.

\section{References}

1. Hawk C, Minkalis AL, Khorsan R, et al. Systematic Review of Nondrug, Nonsurgical Treatment of Shoulder Conditions. J Manipulative Physiol Ther. 2017;40(5):293-319. doi:10.1016/j.jmpt.2017.04.001

2. Castelein B, Cools AMJJ, Parlevliet T, et al. $<\mathrm{p}>$ Prevalence of upper limb pain and disability and its correlates with demographic and personal factors $</ p>$. Cochrane Database Syst Rev. 2019;18(1):1-18. doi:10.1080/17453674.2017.1364069

3. Reijneveld EAEE, Noten S, Michener LA, Cools A, Struyf F. Clinical outcomes of a scapular-focused treatment in patients with subacromial pain syndrome: A systematic review. Br J Sports Med. 2017;51(5):436-441. doi:10.1136/bjsports-2015-095460

4. Littlewood C, Cools AMJ. Scapular dyskinesis and shoulder pain: the devil is in the detail. Br J Sports Med. 2018;52(2):7273. doi:10.1136/bjsports-2017-098233

5. Djade CD, Porgo TV, Vignon Zomahoun HT, Perrault-Sullivan G, Dionne CE. Incidence of shoulder pain in 40-yearolds and over and associated factors: a systematic review. Eur J Pain. 2019;(March):1-12. doi:10.1002/ejp.1482

6. Ellenbecker TS, Cools A. Rehabilitation of shoulder impingement syndrome and rotator cuff injuries: an evidence-based review. Br J Sports Med. 2010;44(5):319-327. doi:10.1136/bjsm.2009.058875

7. Holmgren T, Hallgren HB, Öberg B, Adolfsson L, Johansson K. Effect of specific exercise strategy on need for surgery in patients with subacromial impingement syndrome: Randomised controlled study. Br J Sports Med. 2014. doi:10.1136/bjsports2014-e787rep

8. Page P. Shoulder muscle imbalance and subacromial impingement syndrome in overhead athletes. Int J Sports Phys Ther. 2011;6(1):51-58. http://www.ncbi.nlm.nih.gov/pubmed/21655457. Accessed May 28, 2020.

9. Steuri R, Sattelmayer M, Elsig S, et al. Effectiveness of conservative interventions including exercise, manual therapy and medical management in adults with shoulder impingement: A systematic review and meta-analysis of RCTs. Br J Sports Med. 2017;51(18):1340-1347. doi:10.1136/bjsports-2016-096515

10. Juel NG, Natvig B. Shoulder diagnoses in secondary care, a one year cohort. BMC Musculoskelet Disord. 2014;15(1). doi:10.1186/1471-2474-15-89

11. Camargo PR, Alburquerque-Sendín F, Avila MA, Haik MN, Vieira A, Salvini TF. Effects of Stretching and Strengthening Exercises, With and Without Manual Therapy, on Scapular Kinematics, Function, and Pain in Individuals With Shoulder Impingement: A Randomized Controlled Trial. J Orthop Sports Phys Ther. 2015;45(12):984-997. doi:10.2519/jospt.2015.5939

12. Saito H, Harrold ME, Cavalheri V, McKenna L. Scapular focused interventions to improve shoulder pain and function in adults with subacromial pain: A systematic review and meta-analysis. Physiother Theory Pract. 2018;34(9):653-670. doi:10.1080/09593985.2018.1423656

13. Struyf F, Cagnie B, Cools A, et al. Scapulothoracic muscle activity and recruitment timing in patients with shoulder impingement symptoms and glenohumeral instability. J Electromyogr Kinesiol. 2014;24(2):277-284. doi:10.1016/j.jelekin.2013.12.002

14. Lawrence RL, Braman JP, Laprade RF, Ludewig PM. Comparison of 3-dimensional shoulder complex kinematics in individuals with and without shoulder pain, part 1: Sternoclavicular, acromioclavicular, and scapulothoracic joints. J Orthop Sports Phys Ther. 2014;44(9):636-645. doi:10.2519/jospt.2014.5339

15. Reddy AS, Mohr KJ, Pink MM, Jobe FW. Electromyographic analysis of the deltoid and rotator cuff muscles in persons with subacromial impingement. J Shoulder Elb Surg. 2000;9(6):519-523. doi:10.1067/mse.2000.109410 
16. Pyndt L, Nørregaard J, Dyhre-poulsen P, Winther A. The activity pattern of shoulder muscles in subjects with and without subacromial impingement The activity pattern of shoulder muscles in subjects with and without subacromial impingement. J Electromyogr Kinesiol. 2010;19(5):789-799. doi:10.1016/j.jelekin.2008.08.006

17. Klintberg IH, Cools AMJ, Holmgren TM, et al. Consensus for physiotherapy for shoulder pain. Int Orthop. 2015;39(4):715-720. doi:10.1007/s00264-014-2639-9

18. Harput G, Güney-Deniz H, Düzgün I. Upper to middle trapezius muscle activation ratio during scapular retraction exercise at different shoulder abduction ancles. Fiz Rehabil. 2017;28(3):111-117. doi:10.21653/tfrd.360019

19. Kara D, Harput G, Duzgun I. Trapezius muscle activation levels and ratios during scapular retraction exercises: A comparative study between patients with subacromial impingement syndrome and healthy controls. Clin Biomech (Bristol, Avon). 2019;67:119-126. doi:10.1016/j.clinbiomech.2019.05.020

20. Castelein B, Cools A, Parlevliet T, Cagnie B. Modifying the shoulder joint position during shrugging and retraction exercises alters the activation of the medial scapular muscles. Man Ther. 2016;21:250-255. doi:10.1016/j.math.2015.09.005

21. Skolimowska B, Barczyk K, Dudek K. Bioelectric activity of selected muscle groups in people with impingement syndrome. Acta Bioeng Biomech. 2009;11(May 2014):37-45.

22. Page MJ, Green S, Mcbain B, et al. Manual therapy and exercise for rotator cuff disease. Cochrane Database Syst Rev. 2016;2016(6). doi:10.1002/14651858.CD012224

23. Dong W, Goost H, Lin XB, et al. Treatments for shoulder impingement syndrome a prisma systematic review and network meta-analysis. Med (United States). 2015;94(10):1-17. doi:10.1097/MD.0000000000000510

24. Heron SR, Woby SR, Thompson DP. Comparison of three types of exercise in the treatment of rotator cuff tendinopathy/shoulder impingement syndrome: A randomized controlled trial. Physiother (United Kingdom). 2017;103(2):167-173. doi:10.1016/j.physio.2016.09.001

25. Arias-Buría JL, Fernández-de-las-Peñas C, Palacios-Ceña M, Koppenhaver SL, Salom-Moreno J. Exercises and Dry Needling for Subacromial Pain Syndrome: A Randomized Parallel-Group Trial. J Pain. 2017;18(1):11-18. doi:10.1016/j.jpain.2016.08.013

26. Bury J, West M, Chamorro-Moriana G, Littlewood C. Effectiveness of scapula-focused approaches in patients with rotator cuff related shoulder pain: A systematic review and meta-analysis. Man Ther. 2016;25:35-42. doi:10.1016/j.math.2016.05.337 27. Lewis J, Mccreesh K, Roy JS, Ginn K. Rotator cuff tendinopathy: Navigating the diagnosis-management conundrum. J Orthop Sports Phys Ther. 2015. doi:10.2519/jospt.2015.5941

28. Seitz AL, McClure PW, Finucane S, Boardman ND, Michener LA. Mechanisms of rotator cuff tendinopathy: Intrinsic, extrinsic, or both? Clin Biomech. 2011;26(1):1-12. doi:10.1016/j.clinbiomech.2010.08.001

29. Lunden JB, Braman JP, Laprade RF, Ludewig PM. Shoulder kinematics during the wall push-up plus exercise. J Shoulder Elb Surg. 2010;19(2):216-223. doi:10.1016/j.jse.2009.06.003

30. Muething A, Acocello S, Pritchard KA, Brockmeier SF, Saliba SA, Hart JM. Shoulder-muscle activation in individuals with previous shoulder injuries. J Sport Rehabil. 2015;24(3):278-285. doi:10.1123/jsr.2014-0160

31. Schory A, Bidinger E, Wolf J, Murray L. A systematic review of the exercises that produce optimal muscle ratios of the scapular stabilizers in normal shoulders. Int J Sports Phys Ther. 2016;11(3):321-336. http://www.ncbi.nlm.nih.gov/pubmed/27274418. Accessed October 31, 2019.

32. Cools AM, Declercq GA, Cambier DC, Mahieu NN, Witvrouw EE. Trapezius activity and intramuscular balance during isokinetic exercise in overhead athletes with impingement symptoms. Scand J Med Sci Sport. 2007;17(1):25-33. doi:10.1111/j.16000838.2006.00570.x

33. Martins J, Napoles B V., Hoffman CB, Oliveira AS. Versão brasileira do shoulder pain and disability index: Tradução, adaptação cultural e confiabilidade. Rev Bras Fisioter. 2010;14(6):527-536. doi:10.1590/s1413-35552010000600012

34. Orfale AG, Araújo PMP, Ferraz MB, Natour J. Translation into Brazilian Portuguese, cultural adaptation and evaluation of the reliability of the Disabilities of th Arm, Shoulder and Hand Questionnaire. Brazilian J Med Biol Res. 2005;38(2):293-302. doi:10.1590/S0100-879X2005000200018

35. La AM, Mk W, Wc D. Reliability and Diagnostic Accuracy of 5 Physical Examination. YAPMR. 2009;90(11):1898-1903. doi:10.1016/j.apmr.2009.05.015

36. Ribeiro DC, Castro MP de, Sole G, Vicenzino B. The initial effects of a sustained glenohumeral postero-lateral glide during elevation on shoulder muscle activity: A repeated measures study on asymptomatic shoulders. Man Ther. 2016;22:101108. doi:10.1016/j.math.2015.10.014

37. Toonstra J, Mattacola CG. Test-retest reliability and validity of isometric knee-flexion and -extension measurement using 3 methods of assessing muscle strength. J Sport Rehabil. 2013;Technical(7):1-5. doi:10.1123/jsr.2013.tr7

38. Alpert SW, Pink MM, Jobe FW, McMahon PJ, Mathiyakom W. Electromyographic analysis of deltoid and rotator cuff function under varying loads and speeds. J Shoulder Elb Surg. 2000;9(1):47-58. doi:10.1016/S1058-2746(00)90009-0

39. Kibler BW, Sciascia AD, Uhl TL, Tambay N, Cunningham T. Electromyographic analysis of specific exercises for scapular control in early phases of shoulder rehabilitation. Am J Sports Med. 2008;36(9):1789-1798. doi:10.1177/0363546508316281

40. Michener LA, Sharma S, Cools AM, Timmons MK. Relative scapular muscle activity ratios are altered in subacromial pain syndrome. J Shoulder Elb Surg. 2016;25(11):1861-1867. doi:10.1016/j.jse.2016.04.010

41. Fennell J, Phadke CP, Mochizuki G, Ismail F, Boulias C. Shoulder retractor strengthening exercise to minimize rhomboid muscle activity and subacromial impingement. Physiother Canada. 2016;68(1):24-28. doi:10.3138/ptc.2014-83

42. DiGiovine NM, Jobe FW, Pink M, Perry J. An electromyographic analysis of the upper extremity in pitching. J Shoulder Elb Surg. 1992. doi:10.1016/S1058-2746(09)80011-6 
43. Contemori S, Panichi R, Biscarini A. Effects of scapular retraction/protraction position and scapular elevation on shoulder girdle muscle activity during glenohumeral abduction. Hum Mov Sci. 2019;64(July 2018):55-66. doi:10.1016/j.humov.2019.01.005

44. Forbush SW, Bandy WD, Garrison MK, Graves LC, Roberts R. Testing infraspinatus and deltoid muscles with new technique to decrease deltoid activity during testing using emg analysis. Int J Sports Phys Ther. 2018;13(5):896-904. doi:10.26603/ijspt20180896

45. Sciascia A, Kibler W Ben. Current concepts: Scapular dyskinesis. Br J Sports Med. 2010;44(5):300-305. doi:10.1136/bjsm.2009.058834

46. Mottram SL, Woledge RC, Morrissey D. Motion analysis study of a scapular orientation exercise and subjects' ability to learn the exercise. Man Ther. 2009;14(1):13-18. doi:10.1016/j.math.2007.07.008

47. Larsen CM, Juul-Kristensen B, Olsen HB, Holtermann A, Søgaard K. Selective activation of intra-muscular compartments within the trapezius muscle in subjects with Subacromial Impingement Syndrome. A case-control study. J Electromyogr Kinesiol. 2014;24(1):58-64. doi:10.1016/j.jelekin.2013.09.008

48. Hickey D, Solvig V, Cavalheri V, Harrold M, Mckenna L. Scapular dyskinesis increases the risk of future shoulder pain by $43 \%$ in asymptomatic athletes: A systematic review and meta-analysis. Br J Sports Med. 2018;52(2):102-110. doi:10.1136/bjsports-2017-097559

49. Pribicevic M. The Epidemiology of Shoulder Pain: A Narrative Review of the Literature. In: Pain in Perspective. InTech; 2012. doi:10.5772/52931

50. Facci LM. Síndromes Dolorosas do Ombro: Análise de sua Incidência e Características. Arq Ciências da Saúde da UNIPAR. 2000;4(3). https://revistas.unipar.br/index.php/saude/article/view/1028. Accessed February 27, 2021.

51. Garzedin DDDS, Matos MAA, Daltro CH, Barros RM, Guimarães A. Pain severity in patients with painful shoulder syndrome. Acta Ortop Bras. 2008;16(3):165-167. doi:10.1590/s1413-78522008000300008

52. Hanvold TN, Waersted M, Mengshoel AM, Bjertness E, Twisk J, Veiersted KB. A longitudinal study on risk factors for neck and shoulder pain among young adults in the transition from technical school to working life. Scand J Work Env Heal. 2014;40(6):597-609. doi:10.5271/sjweh.3437 